\title{
CHIU-CHIU 200: POBLADO AGROALFARERO TEMPRANO ${ }^{1}$
}

\section{Maria Antonia Benavente Aninat}

Trabajos acerca del Poblamiento del Período Temprano son escasos en número $\mathrm{y}$, en general, han estado referidos a yacimientos ubicados en la costa; por esta razón hemos creído de interés publicar un informe preliminar sobre el tema.

El interés no surge sólo de la existencia del yacimiento, sino también por la identificación de cerámica aún no descrita para la zona, lo que permite a otros estudiosos del área poder avanzar en nuevos descubrimientos e investigaciones de sitios de este período poco conocido.

El trabajo específicamente se centra en un Poblado Temprano, y es en base a él que nos proponemos los siguientes objetivos:

1. Identificar y caracterizar un complejo cultural agroalfarero temprano.

2. Ubicación cronológica del grupo.

3. Discusión de los datos y formulación de hipótesis.

\section{PRESENTACION DE LA DATA}

\section{Caracteristicas del yacimiento.}

El sitio 200 se ubica en la segunda región, provincia de El Loa, localidad de Chiu-Chiu.

Se encuentra situado en la terraza más alta en la ribera este del río Loa, a 90 metros al norte del Poblado Fortificado de Chiu-Chiu. Limita al cste con el camino de Lasana; al oeste con la ribera este del río Loa; al norte con una pequeña quebrada artificial y al sur con el poblado fortificado.

Desde un punto de vista topográfico pre- senta un leve declive de oeste a este y de sur a norte. Su superficie muestra múltiples irregularidades, compuestas de dos tipos de fosos principalmente:

a) Grandes fosos de formas irregulares que han alterado la superficie primitiva del poblado, $y$

b) Fosos de dimensiones menores, los cuales mantienen un patrón en su forma, sea ésta oval o subcircular, los cuales en su parte exterior presentan montículos de variadas dimensiones, de textura gruesa (basureros), el interior de estos fosos presenta una textura fina, producto del relleno de agentes eólicos.

En lo que respecta a las limitaciones de estudio, presentadas por el poblado, se refieren específicamente a los diversos agentes que han alterado la superficie del mismo, siendo los principales, la preocupación en tiempos prehispánicos (Poblado Fortificado de Chiu-Chiu) y los saqueos sistemáticos que hemos observado en el lugar. A pesar de todo, consideramos que las alteraciones son parciales y están restringidas sólo a un área (central). De este modo pensamos que el sitio con su gran cantidad de estructuras nos dará datos valiosos en cuanto a su funcionamiento.

Los trabajos realizados hasta el momento

${ }^{2} \mathrm{E} l$ presente trabajo es parte de una investigación mayor que está llevando a cabo el autor, con el fin de obtener el grado de Licenciado en Antropolo. gía con mención en Arqueologla y Prehistoria. 
para esta primera etapa se han concentrado a:

a) Levantamiento topográfico del total del yacimiento, lo que arrojó una superficie de $28.693 \mathrm{~m}^{2}$;

b) Cuadriculación total del poblado: la orienración de las mismas están referidas de norte a sur, correspondientes desde un punto de vista matemático al eje de las ordenadas, numeradas en forma correlativa de 1 a. 13; de este a oeste (eje de las abcisas) de la letra A a la M. Esto nos arrojó un total de 169 cuadrículas, con una superficic de $15 \times 15$ metros cada una;

c) Recolección en forma sistemática en cada cuadrícula de los elenientos culturales que había en la superficie del poblado.

\section{Caracteristicas medioambientales}

Los datos que nos entrega el medioambiente podemos sintetizarlos de la siguiente manera:

Se encuentra a una altitud de $2.200 \mathrm{~m}$. s.n.m. con una distancia a la costa de alrededor de $250 \mathrm{~km}$.

Las oscilaciones diarias de temperatura son mayores en verano que en invierno, en que los vientos son fuertes y sccos soplando durante casi todo el año (los vientos constituyen el principal agente modelador del relieve). Las lluvias tienen un carácter estacional, es decir, se ubican en ia época del denominado "invicr. no boliviano". "Las condicioncs topográficas señalan una cuenca lacustre para el sector de Chiu-Chiu, que ha sido vaciado por el sistcma del río Loa. Debido al origen de la cuenca existen en su interior algunos sectores planos con drenaje insuficiente, en los que se han formado vegas, particularmente en el sector sur" (Meneses, 1967: 32).

En estas vegas predominan los pastos cortos y duros que, en conjunto con las gramíneas, en sus diferentes especies, se dan como un ajuste especial para que subsistan principalmente los auquénidos. En general, la vegeta- ción arbustiva está compuesta del jaral desértico (cachiyuyo, brea, rica rica y otros). Las es. pecies mayores son a lgarrobos, pimicntos y chañares.

Dentro de este marco regetal se desarrollan varios tipos de animales, insectos, reptiles, aves, roedores, etc.

"En lo que respecta a los steeios, éstos coresponden al típico suclo gris del cima desértico de la región, carecon del horizonte $A$ y presentan características de durcza y salinidad" (Meneses, 1967: 34).

Aunque postulamos dichos datos como pertenecicntes a la época en que funcionó cl Poblado, debemos mirarlos con cierta reserva, ya que, como todos sabemos, hay un lento. pero progresivo desecamiento del área que cstamos estudiando.

\section{Caracteristicas culturales}

La superficie del poblado se caracteriza por presentar diversos tipos de materiales, numéricamente significativos.

Fuera de algunos rasgos culturales identifi. cados romo pertenecientes al Poblado Fortificado de Chiu-Chiu (corámica) (Thom ss y BE. NAVENTE, 1974-75) más los restos de inhumaciones, el resto de los elementos presentan formas sui géneris y características.

Encontramos abundancia de huesos (auquénidos-roedores), fragmentos de conchas ? vértebras de pescado.

Dentro de los fragmentos de tejidos hemos podido obscrvar algumos con técnica de red, otros al parecer pudieran corresponder al de. nominado "ligimento de cstera" o cntrelazado (Fuentes, 1965: fotos 57 y 633). Otra variedad de tejido que hemos registrado es aque. lla que, usando de vellones de lana se van pasando hilos de pelo humano, los cuales amarran plumas de ave. En este mismo item tambiẻn se presentan hilos torcidos, algunos de lana, otros, al parccer, de vegetales.

Entre lós elementos vegetales se pueden registrar panojas y mazorcas de maíz (pequeñas), semillas de algarrobo. 
El material lítico está representado por: núcleos, perforadores, raspadores, cuchillos, artefactos de molienda (manos de conana, manos de mortero), palas, hachas (pequeñas), las cas y láminas (producto de astillamiento). En general es un material bastante standard en cuanto a técnica y tamaño.

La materia prima empleada es la andesita (instrumentos), cuarcita (perforadores), sílex (puntas) y el granito en su variedad de roca tipo macrogranuda o microgranuda, ha sido utilizada especialmente para la confección de los artefactos de molienda, palas y hachas de mano.

La alfarería se caracteriza por la elaboración de cerámica exclusivamente monócroma, cifé. roja, gris y negra; alcanzando un nivel técnico bajo. El tramiento de superficie se caracteriza por ser alisado, pulido y pintado. El agregado de una decoración, ya sea, incisa, modelada, imbricada, completaría el tratamiento de este complejo cerámico. Otra característica que surge en cuanto a la pasta, es el antiplástico (grande), generalmente de cuarzo y biotita; siendo su distribución irregular, la textura de la misma es apretada, llegando algunas veces a compacta. La cocción es oxidante, en forma irregular, ya que en la mayoría de los fragmentos se nos presenta, en forma constante, el núcleo en la sección de la pieza.

\section{ASPECTOS METODOLOGICOS}

Para el análisis del material lítico, de esta primera etapa, hemos considerado el modelo postulado por Serracino y Thomas (1973:55), en que los autores postulan las siguientes categorias:

- Categoria I: Instrumentos sobre lasca (raspadores-raederas).

- Categoría II: Instrumentos sobre lámina (cuchillos de bisel recto).

- Categoría mi: Instrumentos cuyas líneas de contorno establecen una clara diferencia entre cusrpo y punta (perforadores-buriles).

- Categoría Iv: Instrumentos cuyo extremo distal siguen la línea de simetría del ob. jeto y forma un todo con el cuerpo (puntasj.

- Categoría v: Instrumentos hechos a partir de rodados medianos (manos de morteromanos de conana-núcleos).

Debemos señalar que al analizar detenidamente cada una de las categorias, notamos que algunas son inclusivas de otras. Por ejemplo, las puntas (Categoría iv) son artefactos hechos a partir de lascas o láminas (Categorías I y II) ; los perforadores (Categoría III) son artefactos confeccionados también a partir de lascas o láminas (Categorías I y II).

A pesar de estas deficiencias hemos considerado este modelo aplicable a nuestra pob'ación lítica, ya que tenemos que tomar en cuenta que buscamos un ordenamiento primario del material, es decir, un acercamiento a la realidad en estudio.

De este modo, desde un punto de vista for. mal-cuantitativo, obtuvimos:

1. Instrumentos cortantes, con tisel tipo media luna: $18 \%$.

2. Instrumentos ángulo abrupto: $19 \%$.

3. Instrumentos confeccionados a partir de núcleos: $16 \%$.

4. Perforadores y buriles: $17 \%$.

5. Puntas: $7 \%$.

6. Artefactos de molienda: $18 \%$.

7. Otros: $5 \%$ \% (Ver Lámina I).

De la clasificación cerámica deducimos las siguientes clases:

1. Clase I: Imbricada.

2. Clase II: Incisa.

3. Clase III: Pulida.

4. Clase Iv: Alisada.

5. Clase v: Pintada. (Ver Lámina ı̣). 


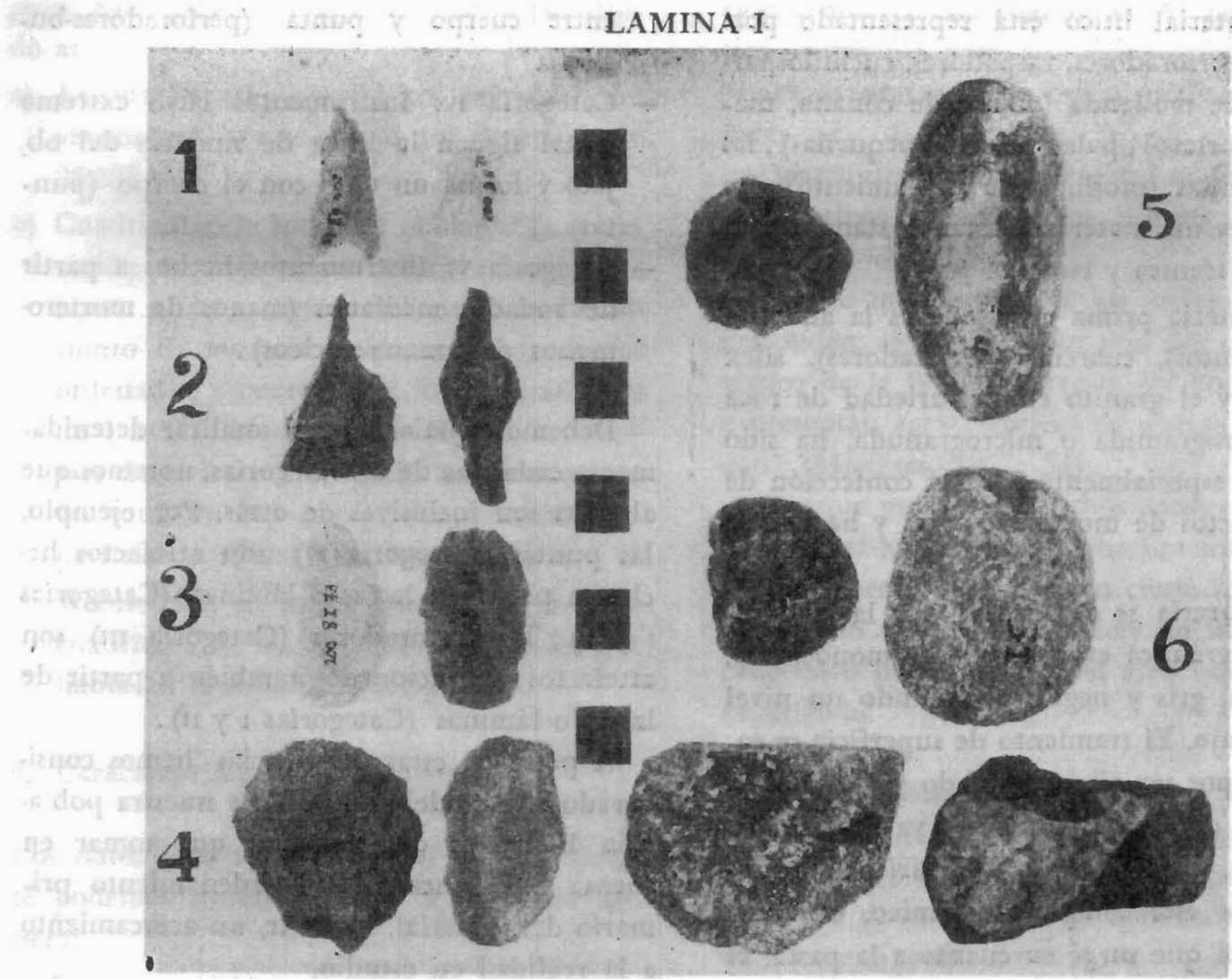

Todas ellas presentan similaridades en:

- Pasta: Tamaño del grano: No uniforme.

Densidad: Fuerte.

Distribución: Irregular.

Textura: Apretada.

- Cocción: Oxidante (en forma predomi. nante).

Irregular.

- Color: Rojo.

Café.

Negro.

Gris.

(Ver Lámina III).

Nos referimos a clases, ya que consideramos que por ser un material de superficie, es necesario, en un trabajo posterior de excavación, verificar su contemporaneidad, tanto con las estructuras, como con el resto del material recogido en el yacimiento, lo que añadirá signi- ficado acerca del funcionamiento del Pobla$\mathrm{do}^{2}$.

Para el resto de los materiales hemos confeccionado una ficha de tabulación, cuyos items principales son los siguientes:

1. Fuentes alimenticias: Vegetales.

\section{Animales.}

2. Especies Industriales: Vegetales. Animales.

3. Elementos de explotación industrial: Caza. Recolección.

Pesca.

\section{ANTECEDENTES BIBLIOGRAFICOS COMPARATIVOS}

Nuestro poblado está constituido por depresiones más o menos hondas, que sugieren la

ఇo hemos considerado los tipos cerámicos pertenecientes al Poblado Fortificado, porque han sido 


\section{LAMINA II}

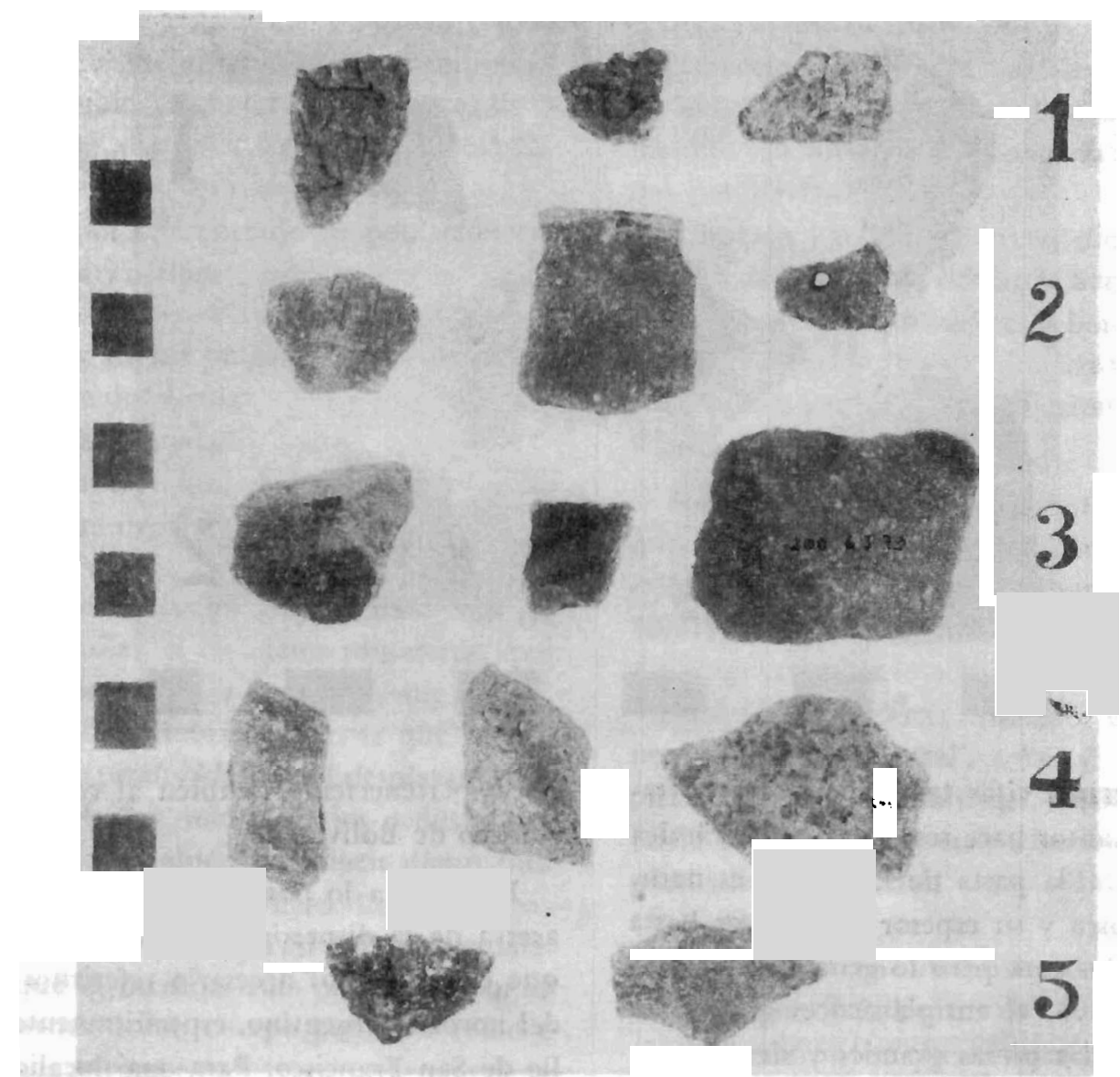

posibilidad de casas semisubterráneas. Por tanto, lo definiremos como formado de habitaciones, de acuerdo a Vivante y Palma (1966, 39), en que cada pozo es toda "vivienda natural con desnivel interior negativo intencional, cuya pared total, en sentido vertical, está formada por más del $50 \%$ de la pared del pozo; y semipozo, a aquellas cuyo porcentaje esté por debajo de esta cifra".

Cronológicamente, por las caracteristicas del material ya expuesto, lo situamos dentro de lo que Dick Edgar Ibarra Grasso (1965), ha denominado "Cultura Megalítica o de los Tells Sur Andinos", para el área boliviana.

Este autor señala, "se nos presenta como siendo la más antigua cultura agricola con

tratados anteriormente $\mathrm{y}$ son fácilmenie distinguibles dentro de nuestro yacimiento. cerámica de la región andina de Bolivia" (Ibarra Grasso, 1965:65). Añade más adelante, a través del análisis del indicador cerámico, que se trata de los primeros vestigios alfareros que aparecen en la región andina de Bolivia, aseveración que extendemos para el d́rea que estamos trabajanclo. Se caracteriza por un rasgo fundamental: "este pueblo conoce bien la cerámica, pero todavia no el ar. to de pintarla, por lo mismo toda su cerámica carece de pintura, por más que es rica en formas, adornos modelados y parcialmente $\mathrm{en}$ adomos incisos" (IbARRA Grasso, 1965: 77) ${ }^{3}$.

El mismo autor se refiere como área de dispersión de estos rasgos cerámicos, el Noroeste argentino y Chile.

${ }^{3} \mathrm{El}$ subrayado es nuestro. 


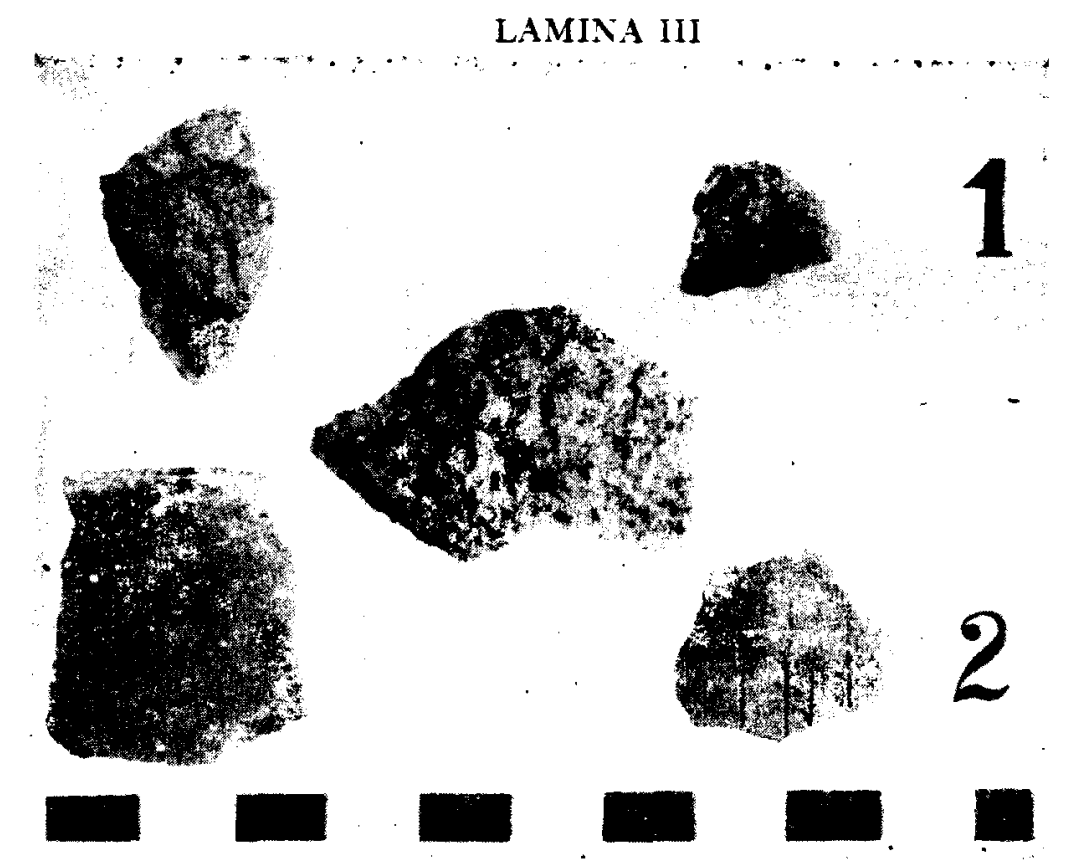

Es interesante citar también la caracterización que cl autor hace respecto de la cerámica temprana ..."la pasta de las piezas es naturamente tosca y su espesor liega a ser hasta cle más de dos cms, pero lo general es de medio a un crn ... el antiplástico es grueso, sobre todo en las piezas grandes y sicmpre vi. sible... el color más generalizarlo es rojizo. pero tambićn hay vasijas grises y casi ricgras... el alisamiento final llega a ser de un pulido no muy fino, ya que siempre se conservan las estrias. y aquí tcnemos mn hecho interesante: cu muchas rasijas pequeñas y medianas, sc ob. nua luna terminación de pulimento hecho come con el dor o de la una, o con un jequeno estoque, se hubiesc pasado en forma objicua po: toda la superficie, on forma de que juedase hicn marado cacla bino de los toques alisaclores... la decoración se presenta como un adorno modelado..." (IbARRA Grisso. 1965: $85-86$ ).

En cuanto al material lítico, comparativamente podemos asociarlo, descle un punto de vista morfológico (puntas confeccionadas con un fino retoque, de base cóncava), a las encontradas en Wankarani, fechado en el 800
A.C.. perteneciente también al complejo megalítico de Bolivia.

Respecto a lo postulado por Ibarra Grasso arerca de la dispersión de dicho complejo, es que consideramo; nocesario refurines al area del noroeste argentino, específicamente al VaJle de San Francisco. Para esa localidad, Serano (1962: 7) postula ..."un antiguo horizonte ccrámico, que hoy mejor calificamos de periodo cultural integradı por varias culurras ... que se habria cxtencliclo en el Noroeste antes del clesarrollo de las culturas agricolas más crolucionaldas".

Serrano señala varias fases para este complejo; resde un punto de rista ergológico comparativo, la lase Fl Infante es lis que presonta mayores similitudes con nuestro material de] sitio 200. El autor describe la cerimica El Infante del siguiente modo: “. . . se trata cle una cerámica tosca, de paredes gruesas... en cuya pasta hay abundancia de gravilla, como si los limos del río no hubieran sido suticientemente limpiados de impurcas... la fabri. cación ha sido hecha por el método de los todetes superpuestos, cuya juntura se observa en muchos casos. También se observan las presiones rítmicas que sobre la superficie interna 
se hizo con los dedos para unirlos ... la superficie interna es bien alisada y pulicla, con lo cual, sin duda, se consiguió impermeabilizar el vaso. En cambio. la superficie externa cs tosca o ligeramente alisada ..." (Serravo. 1962: 62. 24).

El autor ha diferenciado su población cerámica en varios tipos:

1. El Inlante clígito pulgar,

2. El Infunie unguicular.

3. El Infante acanalaclo.

4. El Infantc digitaclo.

5. El Infimte alveolar.

Dentro de los cuales hay aigunos que se pueden asimilar a las clases propuestas por nosotros. Nos referimos a aquellas que presentan la superficic imbricada, en la que su tratamiento fue producido por cl clesplazamiento simultáneo de las yenas de ios dedos y por alveolamiento. también de origen dígito pul. gar, pero cliferente al primero. Lo mismo para la clasc incisa y el tipo El Infante acanala do, en que la incisión fuc producicla por c] deslizamiento de un curpyo punzante romo o más o menos romo.

En relación a los fiem lítico y óseo, existen similitucles con lo registrado en nuestro sitio (núcleos. Jáminas, picelras de mo'er), cpecialnente ch su aspecto técnico. "En los ríos locales esia gente se proveyó de cantos jodarlos que emplearon como percutores o manos do mortero. En algunos se observa el clesgaste de sus cxtremos por continuado golpoteo de percusión y tambićn el desprendimicn10) de pequcinas lascas. Cuando el ranto roclado fue empleado como núcleo, el continuado desprendimiento de lascas origiun superficics de varias caras de percusión" (SgrRano. 1962: 28).

En cuanto a forma y funcionalidad, tencmos las hachas, percutores, cuchilics, morteros y puntas.

Las puntas le base escotada y de un tamaño que varía de 4 a $6 \mathrm{~cm}$, son similares a las de procedencia boliviana (Wankarani), fecha- clo tempranamente con $\mathrm{C} 14$ en el 800 A.C. (Ponce Sanginés, 1970: 29-31).

Respecto a los tejiclos (tócnica de red y tipo entrelazado com plumas de are y pelo hit. mano). son considerados comn rasgos tempra nos por Rivera (1976).

Tanbicn hacenos notar las similitudes, on razón a la naturaleza di:l sitio: Serrano señala que parecen sei equivalentes a bordos o asientos de habitación, en que ios fogones inclican ocupaciones sucesivas de poblaciones que iban y venían.

Referente a la bibliografía que trata sobre nuestro territorio, especificamente parat la localidad que estamos estudiando, Pollard (1971) se reficre, vagamente. sobre if Poblato. Lo sitúa cronológ camone on lo gule él denomina "Complejo Vega Alta u"; entendiendo por romplejo cultural... "total of cultural forms (artifact. assemblage. settlement pat tems, food cconomy, ctc.) characteristics of a particular region and time span" (Pollard, 1971: 14).

Lo describe con una población estimada de 40 a 80 personas, dentro de un área de 100 por 200 metros, en que la misma área ha sido utilizada como un comenterio con características cerámicas tipo Lasana 111, el que laa sido totalmente destruiclo. Así hay muy pocas probabilidedes de determinar si las estructuras es. tuvicron originalmente asociadus con el componcute Vega Alta 11. Respecto al mucrial lítico, cl autor señala que se han recolectado alreded or de 40 manos y 10 conamas partidas lo que sugiere una ocupación relativamente sustancial.

Aunque debemos considerar que el trabajo cle Pollard está referido específicamente a una prospección áreal, sus conclusiones en mu chos aspectos son valiosos; con respecto al silio que estamos trabajando, debemos señalar las siguientes deficiencias:

1. El yacimiento sólo ha sido parcialmente destruido (cementerio y saqueo está referido sólo a la parte central), por tanto, la mayoría de las estructuras son posibles de 
identificar en todos sus aspectos (forma y basureros);

2. En cuanto al número de personas que pudieron ocupar el poblado, acotamos que es imposible estimar, sin métodos apropiados, los aspectos demográficos de un yacimiento arqueológico;

3. Relativo a la cantidad de material superficial, éste es mucho mayor que la cantidad que scñala el autor; el cual debió haber prospectado sólo parte de la superficie total del poblado.

Después de analizar en forma exhaustiva el artículo, consideramos que nuestra data está referida especificamente a lo que el autor de- nomina Complejo Vega Alta I o Temprano, caracterizado por... "open camusites yieíded coarse temper sherds from cooking and storagc jars. stemmed and concavc, base proyectil points, microperforators, stone flake knives, scrapers, notched tools, irregular cores, stone and skell beads, and retouched utilized, and waste flakes. Also present in the assemblage are ovoid and trapezoidal tabular porphyritic rocks, bifacially flaked on the edges... mortars and pestles... The lithic artifacts reflect the use of several stone typoes: cherts, malachite, silicified tuffs, quarzite, felsites, and basalts, including small quantities of obsidian. The cherts and tuffs are found locally, bu1 the other materials had to have been brougth from highland regions to the east or nortll, a distance of $50-75 \mathrm{~km}$. Small quantities of seashell are also present, apparently traded in from the coast, at least $200 \mathrm{~km}$ distant ... subsistence is likely to have centered on wild guanaco and vicuna, supplemented with various plant resources. Edidle rhizhomes, common in late preceramic midclens, and algarrobo pods may have been ground on the milling stone and mortars" (Pollard, 1971: 44-45).

Para el área de San Pedro de Atacama, Gustavo Le Paige (1974) hace referencia para Sequitor Alambrado Oriental, de un sitio habitacional en que la cerámica presenta "varios tipos primitivos anteriorcs al rojo pulid". siendo fechado por C 14 en Argentina alrededor del 800 A.C., perteneciente a la cultura San Francisco" (Le PAige, 1974: 102)

Reynaldo Lagos hace referencia para Que. brada Tambores, localidad de Río Grande, de otro poblado (pronto a trabajarse), con las mismas características del sitio 200 , lo que estaria confirmando un área de dispersión bien delimitada y una contemporaneidad cronológica, que confirma lo comunicado por Carlos Thomas, refiriéndose al sitio 200 que "encontró cerámica prinitiva con el complejo lítico de Chiuchiu. Esta misma cerámica es igual a aquella de San Pello de Atacama con pasta de granos gruesos de cuarzo y otros minerales" (c.f. Serracino, 1974: 108).

Basándonos en la data presentada nos planteamos las siguientes interrogantes:

1. ¿Fue muy diferente el entorno biofísico del que conocemos hoy?

2. Si manturo sicmprc fuertes limitaciones parecidas a las actuales. ¿cómo influyó er, cl comportamiento de la población?

3. ¿Cuál fue el tamaño de la población?

4. Si fue de una alta clensidad, sería una de las razones que rondujo al grupo a tener una gran movilidarl:

5. Sabemos por la data que núcleos de población en el altiplano, con caracteristicas similares ocupan tempranamente nichos ecológicos más favorables:

¿Qué motivó a cstos grupos a trasladarse a regiones que, como nosotros apreciamos, son marginales en cuanto a situación y menos favorables en relación a los recursosi?

6. ¿De qué tipo fue esta movilidad? ¿Producto de comercio? intercambio? ¿Colonización de nuevas áreas?

7. ¿Dónde amplió su raclio de acción? : $\mathrm{Ha}$. cia áreas cordilleranas? ¿Qué relaciones mantuvo entonces con la costa?

8. Debido a la movilidad, jadecuó el grupo su tecnologíar, ¿la economía especializó sus elementos?

9. ¿Qué tipo de asentamiento tuvo el grupo? 
¿Permanente?, ¿estacional?, ¿̨campamentos base?, $y$

10. ¿Qué organización tenía el grupo? :lgualitaria?, ¿diferenciada en clases?

Postulamos desde un punto de vista general que el medio ambiente debió haber tenido características similares a las que prevalecen actualmente en el lugar. Hay dos hechos que nos permiten aseverar esto:

1. El emplazamiento del poblado sobre un solevantamiento calcáreo, con el fin cle mantener las zonas de vegas (norte y cuenca del Loa) libres para utilizarlas como pastos para el ganado, áreas de recolección, cultivos rudimentarios.

2. Dentro de nuestro registro ergológico (flora-fauna), producto de la recolección, encontramos que las especies no han variado mayormente (algarrobo, brea, chilca, molle); podríamos sí encontrar cambios en cuanto a la fauna, pero éstos se deben a la constante depredación por parte del hom. bre en la zona.

La presencia de este grupo en nuestro territorio se debe, posiblemente, a presiones demográficas en el altiplano, prolucto de un adelanto tecnológico mayor (Wankarani-Chiripa-Tiwanaku I), lo que condujo forzosamente a migraciones masivas a áreas aledañas, en busca de nuevos asentamientos.

Los nuevos asentamientos ubicados en áreas de débil potencial ecológico, necesariamente los ha conducido a aprovechar pasturas en la cordillera. Si planteamos este tipo de condiciones, el grupo debió necesariamente hraberse movilizado con su ganado a las zonas altas en el verano, con el fin de obtener mayores recursos para sus animales, bajando a las vegas en épocas del denominado invierno boliviano.

En relación a la presencia de ciertas especies marinas (conchas), éstas se deben, por su cantidad minoritaria, a producto de intercambio esporádico con grupos de economía marítima.
Considerando ia procedencia de lit materia prima con la cual se confeccionó el material lítico, ni la diorita, el granito, cuarzo y el sílex son materiales que se encuentren en abundancia en la localidad; por tanto, este grupo clebió haber buscado dichos recursos del subsuelo fuera del área señalada.

La funcionalidad de los instrumentos está directamente relacionada con una actividad preponderante, el beneficio de animales. Porque si pensanos que los cuchillos (instrumentos de bisel recto) cortan carne; raspadores: raspan cueros; las raederas: descarnan; perforadores y buriles sirven como instrumen. tos punzantes para perforarlos y su posterior elaboración; las puntas para cazar especies conıo guanacos, vizcachas, animales menores, la funcionalidad es clara.

Resulta cierto también que esta gente utilizaba el área de su poblado, ejemplo concreto lo tenemos al registrar grandes cantidades de brea, plumas de pato, algarrobo, chilca, todos recursos obtenibles en el lugar.

Pero dicho grupo no sólo mantenía una actividad ganadera, sino recolectora a la vez, ya que encontramos artefactos de molienda representados también en forma mayoritaria. Este aspecto debemos complementarlo posteriormente con los restos de semillas, en general de vegetales, que obtengamos por medio de excavaciones.

\section{ASPECTOS TEORICO METODOLOGICOS}

Si consideramos que las hipótesis expuestas con anterioridad ofrecen muchas de ellas para su verificación un alto grado de comple. jidad; algunos problemas, tales como la organización social y política del poblado, entre otros, requerirán de una acuciosidad mayor en su tratamiento. Pensamos, por tanto, que en un futuro próximo y con un mayor conocimiento empírico a través de una Teo. ría Ecológica Específica, la denominada "Pa. trones de Asentamiento", podrán darnos una solución más próxima a la reahiad. 
Este enfoque toma cono unidades básicas de análisis:

1. La habitación o estruccura incividual;

2. La disposición de la comunidad, y

3. La manera en la cual las comunidades pertenecientes a una cultura, sociedad, se distribuyen en el paisaje.

Los patrones dispucstos en cada uno de estos niveles pueden verse funcionalmonte relacionados a todos los aspectos de una cultura; además, arrojan luz sobre una variedad de problemas. Pero, en el hecho, carla nivel desarrolla tendencias especialniente apropiaclas al estudio de los aspectos particulares de la sociedad.

La estructura individual arroja información acerca de la organización atesanal y quizás su importancia relacionaclia a los diferentes aspectos de la estructura social; el plan de la comunidad ha proporcionado hitil información acerca de la organización de los linajes y la adaptación de lis comunidades a sus medios culturales y físicos; los patrones zonales reflejarán los aspectos políticos, sociales, comercio y guerra.
Lo mismo cabe señalar clesde un ánguiv metodológicoticnico. A este respecto, y debido a la naturalesa misma clel yacimiento (tamano $y$ densidad de estructuras habitacionales), creemos conveniente aplicar la técnica de antílisis espacial postulada por Robert Whallon (1973) .

El análisis dimensional de variancia ha si. do aplicado en el campo de la coología y está refericlo al análisis cle patrones, utilizando una malla de cuadrados contiguos. Es, en general, la lorma más común y simplificada para detectar y definir espacialmente las concentraciones en la data expresada en números para cada midad de malla (grid).

Con el lin de obtener dalos acerca del agregado humano que ocupó el poblado, aplicaremos la técnica postulada por Cook y Treganza (1950). Nos referimos específicamente a la relación existentc entre el área total de estructuras habitacionales y cl número posible de habitantes que lo oeupó.

Aun cuando está la limitación de que el sitio ha siclo remorido en una medida signilicativa, quizás haciendo un examen del tamaño y densidad de los basurcros (contenido proteico), podremos ajustar más el análisis demográfico anteriormente postulado.

\section{BIBLIOG R IFIX}

Cook, S, and Treganza, A. The quantitative in. vestigation of indian mounds. (En: Publication in American Archaelogy an Etlınology, Berkeley. I: of California, 1950, $\left.40, N^{2} 5\right)$.

Druss, Mark. Medio Ambiente, economia de subsistencia y patrones de Asentamicuto del Complejo Chiu-Chiu. (CA 3000 a 2000 A.C.), Norte de Chile. (En: Estudios Atacameños. San Pedro de At:1. cama. Universidad del Norte, 1976, N 4, PP. 17 23) .

Ibarra Grasso, Dick Edcar. Prehistoria de Bolivia. La Paz, ed. Los Amigos del Libro, 1965, Cap. II.

Le Patge, Gustayo. Sequitol Alambrado Oriental. Informes de trabajo. (En: Fstudios Itacameños. San Pedro de Atacama, Iniversidad del Norte. 1974, No 2, P. I04)
Meneses, C. Problemas Geográficos para el desarrollo de Chiu-Chiu y Lasana. (En: Boletín de la Asociación de Cécógrafos de Chile. Ob. cit., 1!67, Arios $1, x ? 3)$.

Pollard, Gordox C. Cultural change and adaptation in the central Atacama - Desert of North. ern Chile. (En: Sawpa Pacha, Berkeley, 1971, No 9, pp. $4(-64)$.

Poxa: Shycines, Crolos. Wankarani y Chiripa, y su relación con Tiwanaku. La Paz, Academia Na. cional de Ciencias de Bolivia, 1970.

Serracino, G. y I homirs, C. Excavación del yaci. miento Confluencia I. (F.n: Boletín de Prehistoria de Chile, Santiago, Depto. de Ciencias Antropologicas y Arqueológicas, $U$. de Chile, 1971, No 4, pp. 49.68) 
Serracino, Grorge. Nuevas fechas de Radiocarbón 14. Noticias. (En: Estudios Atacamcios. San Pe dro de Atacanta, L. del Norte, 1974, X.? $p$. 108).

Serracino, Georce. Tulor 4: 4 pozos de sondeo. (En: Estudios Atacameños, San l'edro de Atacaina, $\mathrm{C}$. del Norte, 1976, N? 4. Pp. 24-31).

Serrano, Antonio. Investigaciones Arqueológicas en el Valle del ró San Francisco. Salta, Fscuela Tipográfica del Colegio Salesiano "Angel Zerda": 1962.

Thomas, C. y Benavente, A. Proposición de un modelo para el análisis de fragmentación cerá. mica poco diagnóstíca. (En: Boletín de Prehistoria de Chile. Santiago, Depto. de Ciencias Antro. pológicas $y$ Arqueológicas, L. de Chile, 1974.75. hños 6.7, N.os $7 \cdot 8$, pp. 39-58).

Trigcer, Brlce, The determiniants of Settlement Patterns. (En: Settlement Archaelogy. Palo Alto California, editeit by K. C. Clang, 1968, pp. 53. 57 .

Viyante, armando y Palda, Nésror. Habitacio. nes pozo y semipozo con paredes de guano en la I'una Argentina. (En: Revista del Museo de La Plata. La Plata, Sección Antropologia, 1966, Iono 11. PP. $17-43)$.

Whallon, Robert. Spatial Analysis of occupation floors 1: Application of dimensional analysis of va. riance. (En: American Antiquity, July 1978, vol. 38, No 3, pp. 266-278). 